\title{
Fabrication of 3D microstructure array on chip for rapid pathogen detection
}

\author{
Kant, Krishna; Anh, Tien Ngo; Matteucci, Marco; Wolff, Anders
}

Published in:

Sensors and Actuators B: Chemical

Link to article, DOI:

10.1016/j.snb.2018.11.008

Publication date:

2019

Document Version

Peer reviewed version

Link back to DTU Orbit

Citation (APA):

Kant, K., Anh, T. N., Matteucci, M., \& Wolff, A. (2019). Fabrication of 3D microstructure array on chip for rapid pathogen detection. Sensors and Actuators B: Chemical, 281, 774-782.

https://doi.org/10.1016/j.snb.2018.11.008

\section{General rights}

Copyright and moral rights for the publications made accessible in the public portal are retained by the authors and/or other copyright owners and it is a condition of accessing publications that users recognise and abide by the legal requirements associated with these rights.

- Users may download and print one copy of any publication from the public portal for the purpose of private study or research.

- You may not further distribute the material or use it for any profit-making activity or commercial gain

- You may freely distribute the URL identifying the publication in the public portal 


\section{Accepted Manuscript}

Title: Fabrication of 3D microstructure array on chip for rapid pathogen detection

Authors: K. Kant, T.A. Ngo, M. Matteucci, A. Wolff

PII: S0925-4005(18)31949-X

DOI: https://doi.org/10.1016/j.snb.2018.11.008

Reference: SNB 25606

To appear in:

\section{Sensors and Actuators $B$}

Received date:

3 July 2018

Revised date:

9 October 2018

Accepted date:

1 November 2018

Please cite this article as: Kant K, Ngo TA, Matteucci M, Wolff A, Fabrication of 3D microstructure array on chip for rapid pathogen detection, Sensors and amp; Actuators: B. Chemical (2018), https://doi.org/10.1016/j.snb.2018.11.008

This is a PDF file of an unedited manuscript that has been accepted for publication. As a service to our customers we are providing this early version of the manuscript. The manuscript will undergo copyediting, typesetting, and review of the resulting proof before it is published in its final form. Please note that during the production process errors may be discovered which could affect the content, and all legal disclaimers that apply to the journal pertain. 


\section{Fabrication of 3D microstructure array on chip for rapid pathogen detection}

K. Kant ${ }^{a \neq^{*}}$, T.A. Ngo ${ }^{b \neq^{*}}$, M. Matteuccib and A. Wolffa

${ }^{a}$ Department of Micro- and Nanotechnology, Technical University of Denmark, $2800 \mathrm{Kgs}$, Lyngby, Denmark

baboratory of Applied Micro and Nanotechnology (LAMINATE), National Food Institute, Technical University of Denmark, 2800 Kgs, Lyngby, Denmark

*Address correspondence to: K. Kant (e-mail: krkant@nanotech.dtu.dk, krishnakant696@gmail.com); T.A.Ngo (e-mail: tinan@food.dtu.dk)

${ }^{\ddagger}$ K. Kant and T. A. Ngo contributed equally, and both should be considered as first authors.

Highlights of the Research Paper:

- An advanced process of free angle photolithography (FAPL) is used for making 3D supercritical angle fluorescence (SAF) structures and transfer them to the polymeric chip by injection molding for low-cost microfluidic devices with the embedded optical sensing. These 3D structures are used for enhancement of fluorescent signal through the unique properties of SAF. Solid phase polymerase chain reaction (SP-PCR) on these SAF structures permits for on-chip pathogen detection. These 3D structures have the 
potential to be widely used in microfluidic chips as a tool for signal enrichment and lowcost point of care systems for optical detection.

- The intensity of the signal vs. probe concentration measured from the rear face for different size of SAF structures and defined the LOD $(0.05 \mathrm{nM})$ of the smallest SAF structure. The use of such small size of SAF structure will enable us to use less volume $(220 \mathrm{pL})$ and save expensive primer and molecular probes in an experiment. The presented smaller SAF have great potential to be used for the multiple detections in a small area with high sensitivity and large field of view which can help to use such chip without a sophisticated optical system. This will allow us to make a cost-effective experimental design with a low volume of the sample and optical readout system. In future development, a lower detection limit could be achieved by reducing the background signal and by using higher wavelength fluorophores. The combination of SP-PCR and L-SAF array would be widely used as a high-throughput biosensor to analyze food, clinical and environmental samples.

\section{Abstract}

An advanced process of free angle photolithography (FAPL) is used for making 3D supercritical angle fluorescence (SAF) structures and transfer them to the polymeric chip by injection molding for low-cost microfluidic devices with the embedded optical sensing. The FAPL was performed via a motorized stage to control the angle of incidence of light and achieve the 
desired shape with dimension from $50 \mu \mathrm{m}$ to $150 \mu \mathrm{m}$ and slope required for the 3D optical structure. These 3D structures are used for enhancement of fluorescent signal through the unique properties of SAF. The presented SAF structure has a reduced active area $(50 \mu \mathrm{m})$ that allows enrichment of the fluorescence efficiency and reduces the amount of sample required for detection on the polymeric microfluidic chip. Herein, we are presenting reduced dimension of SAF structures, fabricated by FAPL process and increases the number of SAF per $\mathrm{mm}^{2}$ area. This also reduced the volume of sample required per test. Improvement in the limit of detections (LOD) is observed when using the small dimensions of SAF. Solid phase polymerase chain reaction (SP-PCR) on these SAF structures permits for on-chip pathogen detection. These 3D structures have the potential to be widely used in microfluidic chips as a tool for signal enrichment and low-cost point of care systems for optical detection.

Keywords: 3D microstructures array, free angle lithography, Microfluidic Chip, Solid phase PCR, Pathogen detection.

Mohammad-Ali Shahbazi 


\section{Introduction}

In recent years various polymer lab-on-a-chip systems have been produced and commercialized with the final goal of obtaining systems that are disposable and compatible with large-scale production [1]. Cyclic olefin copolymers (COCs) are an extremely attractive for large-scale production [2,3]. COC have high optical transparency, chemical inertness, and high heat resistance [4-7]. Before making a disposable chip mold, one should first think of making a suitable quality (smoother surface and easy to replicate) mold for fabrication of chip. Methods such as micro milling, 3D laser cutting and laser milling are developed to fabricate molds for a costeffective polymeric chip. Among these technologies, micro milling and laser milling are widely used to make the molds, and injection molding is used to make the positives replica of those molds. Role of the master mold has strongly been highlighted to achieve defect-free polymer chips in injection molding process $[8,9]$. Past years, studies took place to make a better mold with low surface roughness and defects in the molds to achieve better, smoother and reproducible polymer chip [10]. The micro milling is conventionally used to make a rapid mold for injection molding. However, it cannot achieve lower surface roughness as can be achieved by photolithography. Though, molds made by conventional photolithography process also having some defects or changes of microstructures due to the imperfections in the photolithography process and photoresist $[11,12]$. These defects in structures cause problems during the molding and demolding of the polymer chips. To reduce the surface defects, which are created 
during micro milling, and defects during the conventional photolithography process, we demonstrated the use of FAPL process to produce 3D structures with smoother surface and fewer defects. Using FAPL process allows us to precisely transfer microstructures to Ni master mold and then to the polymeric chip.

In our previous work, we presented the fabrication of supercritical angle fluorescence (SAF) microstructures in polymer by means of injection molding with a micro-milled stamp (here referred as shim) using micro-milling method [13]. The advantage of using injection molding is two-fold: the reproducibility of structures for Lab-on-chip applications with dimensions of tens of nanometers, together with a technique that is ideal for production of large number of samples [14]. The advantage of using SAF structures can be explained if we consider a fluorophore molecule available on the front of a generic surface of a microstructure: for a generic structure, most of the emitted light onto the material is refracted outside the structure and so a very little amount of light passes through to give a signal $[15,16]$. Collection of this unused part of light by a structure that exhibits a supercritical angle of reflection delivers an enhancement in the signal intensity up to 46 folds [13]. Usually this refracted fluorescent light is lost or not been gathered when using a flat surface (microscopic polymer slide) for the signal capturing. Moreover, the SAF structures also offer a great field of view for more efficient signal collection.

In this paper, we introduce the FAPL fabrication process and demonstrate the possibility of achieving lithography made SAF (L-SAF) structures with lower surface 
roughness and higher optical efficience respect to the our previously described micromilled SAF (M-SAF) [13]. Particularly, we present a FAPL fabrication procedure (Figure 1A) for L-SAF structures of different sizes $(50 \mu \mathrm{m}, 100 \mu \mathrm{m}, 150 \mu \mathrm{m})$ and demonstrate their advantages over the SAF structures obtained by injection molding of micro-milled shims. Furthermore, reduction in the dimensions $(50 \mu \mathrm{m})$ of SAF both improves the number of available detection site per area and reduces the required sample volume. We also consider defining the efficiency of the fluorescent signal against the number of molecules. The SP-PCR was performed using DNA probes on the L-SAF array $[17,18]$ (Figure 1B) for the purpose of on-chip pathogen detection. Our experimental outcomes confirmed that by using such kind of SAF arrays, the limit of detection (LOD) can be improved up to $0.05 \mathrm{nM}$ corresponding to $6.62 \times 10^{3}$ molecules. The LOD is calculated by data obtained from a high-end fluorescent scanner. The presented work is strategic in order to have effective multiple on-chip detection sites per $\mathrm{mm}^{2}$ area. Furthermore, fabrication and incorporation of these L-SAF arrays on the chip can easily be done by injection molding for mass production. This also adds-on in the direction of the portable, high-throughput bio-sensing system that can be ideal for onsite diagnosis.

\section{Materials and Methods}

\subsection{Fabrication of SAF structures}

To achieve L-SAF structures by FAPL method, the initial fabrication process was performed with SU-8 from Microchem to check the possibility of fabricating SAF 
structures and Ni molds with an SU-8 template. But after Ni electroplating, the SU-8 structures that remain trapped in the mold after Si etching in $\mathrm{KOH}$; even if after 15 minutes there was an evident removal of a large percentage of the resist from the micro cavities, it was never possible to obtain a full removal of SU-8 residuals. To remove the SU-8 after $\mathrm{KOH}$ etching, an attempt to burn the resist was made. The samples were put into a furnace (resist Pyrolysis furnace, ATV) and heated to $500{ }^{\circ} \mathrm{C}$ for 15 and 25 minutes (Figures S1 supplementary) $[19,20]$. Since SU-8 presented the issue of removal from shim and long processing times, a new negative tone photoresist THB $151 \mathrm{~N}$ from JSR Co. was used in the fabrication of $\mathrm{Ni}$ shim for injection molding of SAF structures. The main advantage of THB $151 \mathrm{~N}$ is that the fabrication processes does not require a postexposure bake and that it easy to remove after the Ni electroplating step. This makes THB 151N preferable over SU-8 for integration in production-like processes [21].

At first, the photoresist was spin-coated at $1200 \mathrm{rpm}$ on a Si wafer and soft baked for 3-5 min to obtain a resist layer of about $50 \mu \mathrm{m}$ thickness. To achieve the higher thickness for 100 and $150 \mu \mathrm{m}$ SAF structures the spin coating step was repeated 2 and 3 times. A final resist thickness of $100,150 \pm 5 \mu \mathrm{m}$ was achieved. We chose such thickness since the goal was to obtain structures with an aspect ratio of about 1 which is ideal for injection molding [22]. The complete fabrication process is described schematically in Figure 2. The FAPL process was executed on a Si wafer with a mask pattern of round holes with diameters ranging from 50 to $200 \mu \mathrm{m}$ using hard contact mode. Such mode was chosen since a gap between resist and mask combined with the rotation would have 
caused undesired widening of the structures. For the same reason, the thickness uniformity of the resist over the whole wafer needs to be controlled. Edge bead removal was thus performed on wafers before the exposure to obtain a thickness uniformity under $\pm 2 \mu \mathrm{m}$ over the 4-inch wafer area. The wafers were then loaded onto the tilting stage, fixed at a given tilt angle and rotated clockwise at $15 \mathrm{rpm}$ and a dose of 1500 $\mathrm{mJ} / \mathrm{cm}^{2}$ was delivered throughout the UV exposure. The resist refractive index, and the request of a 60-degree tilt on the final SAF structures lead to the choice of a fixed tilt angle $\theta$ rot $=52.3$ degrees. After the UV exposure, the wafers were developed automatically (Gamma Cluster System 233, SussMicroTech) alternating 10 puddles of AZ726 MIF developer 60 seconds long with rinsing in deionized water before final drying (Figure 2B) (conditions for all size $50,100,150 \mu \mathrm{m}$ of L-SAF spin speed, exposure time and development time is available in supplementary table S2). A metal $\mathrm{Cr} / \mathrm{Au}$ seed bilayer was then sputtered $(\mathrm{Cr} / \mathrm{Au}=20 / 60 \mathrm{~nm})$ together with a $1 \mu \mathrm{m}$ thick Al sacrificial layer (Kurt J Lesker). The Ni electroplating (Microform.200, Technotrans) was carried out with 3 steps by increasing the current density to minimize the residual stress.

\subsection{Fabrication of master mold and transfer of L-SAF array to polymeric chip}

As a result, the Ni layer was electroplated to a thickness of around $340 \mu \mathrm{m}$ over the sputtered microstructures the fabricated microstructures. After etching the Si (and the Al) in a $\mathrm{KOH}$ bath at $80{ }^{\circ} \mathrm{C}$ (Figure $2 \mathrm{C}$ ), the $\mathrm{Ni}$ shim was then cut with a hydraulically activated system and installed inside the injection molding machine. The characterization at each step of the process is performed by using either scanning 
electron microscopy (SEM, Quanta $200 \mathrm{FEI}$ ) or optical microscopy. For injection molding, TOPAS 5013L COC (Heat Deflection Temperature, $\mathrm{HDTg}=130^{\circ} \mathrm{C}$ ) pellets were purchased from TOPAS Advanced Polymers (Germany) and dried in the hopper of the injection molder (ENGEL Victory 80/45 Tech) (airflow at $90^{\circ} \mathrm{C}$ ) to remove extra moisture. The temperature of the injection molding cylinder was gradually increased from $250{ }^{\circ} \mathrm{C}$ as the polymer exited the hopper to reach $300{ }^{\circ} \mathrm{C}$ at the nozzle. The molten COC was injected into the mold with a speed of $21 \mathrm{~cm}^{3} / \mathrm{s}$ and a pressure of 600 bars. The mold temperature was varied (Variotherm process) from $155{ }^{\circ} \mathrm{C}$ at the moment of injection to $103{ }^{\circ} \mathrm{C}$ when the demolding was performed. In addition, a packing pressure of injection molding ranging from 600 bars was applied for $6 \mathrm{~s}$ injection molded to produce the optics to be introduced into the microfluidic device. The final chips had the dimensions of a microscope slide $(76 \mathrm{~mm} \times 25 \mathrm{~mm} \times 1 \mathrm{~mm})$. The chip has eight parallel arrays located at the center of the chip with a pitch of $6 \mathrm{~mm}$. Each array contains 600, 300 and 100 SAF for $50 \mu \mathrm{m}, 100 \mu \mathrm{m}$, and $150 \mu \mathrm{m}$ respectively. The dimensions of the SAF structures are illustrated in Figure 3.

\subsection{Spotting on SAF structures}

The precise spotting on each SAF structure in an array was performed by a SCiFLEXARRAYER S5 (Scienion, Germany). A microtiter plate was used to keep the serial dilution ( $1 \mu \mathrm{M}$ to $1 \mathrm{pM}$ ) while a glass tip (type 1 ) was taking the solution and washing tip each time before and after changing the sample for spotting. This process is automated and controlled by the software developed by Scienion. The BioAnalyzer $4 F / 4 S$ scanner 
with 400-ms shutter time (LaVisionBioTec $\mathrm{GmbH}$, Bielefeld, Germany) was used for scanning and analyzing spot intensities after spotting all size of SAF structures (Figure S2S4 supplementary). The sample solution containing Cy3 labeled poly(T)poly(C) oligonucleotide in $5 x$ standard saline citrate (SSC) buffer and $0.004 \%$ Triton $X$. The spotting volume is $220 \mathrm{pL}$ was spotted on the front surface of each SAF structure using a SCIFLEXARRAYER S5. The amount of volume is different for different size of the SAF structures to cover all the front face. All comparison experiments among the different size of SAF are conducted with constant volume $220 \mathrm{pL}$. However, this volume is only sufficient for small size $(50 \mu \mathrm{m})$ of SAF to cover all the front face. To cover the front face of 100 and $150 \mu \mathrm{m}$ SAF structures we need 450 and $680 \mathrm{pL}$, respectively.

\subsection{Solid phase PCR on lithography SAF}

\subsubsection{DNA preparation}

Streptococcus pneumonia was from culture collection of National Food Institute, Technical University of Denmark (DTU-Food). S. pneumonia genomic DNA was isolated using DNeasy Blood and Tissue kit (Qiagen, Germany) as instruction from the supplier. The DNA concentration was determined by Nano drop 1000 (Thermo Scientific, USA) and the DNA preparation was stored at minus $20^{\circ} \mathrm{C}$ before use.

\subsubsection{Primers}

To make a model to confirm the performance of SP-PCR on FAPL-generated SAF structures for pathogen detection, we selected a set of primers to amplify pneumolysin 
gene for Streptococcus pneumonia detection [23]. Forward and Cy3-labelled reverse primers were used for the liquid phase amplification. The surface primer was designed as an internal primer and modified at the $5^{\prime}$ end with a poly $T(10)$ and $C(10)$ tail to immobilize on the front of SAF. All PCR primers were synthesized and purchased by DNA technology (Aarhus, Denmark) and the sequences are listed in Table S1 (Supplementary data).

\subsection{Immobilization of surface primers}

The $50 \mu \mathrm{M}$ of surface primers were prepared in a solution containing $5 \times$ SSC and $0.004 \%$ Triton X, and spotted on front of SAF structure inside the chamber of a polymeric (COC) by sciFLEXARRAYER. After spotting, the microchip was dried and treated with UV irradiation at the wavelength of $254 \mathrm{~nm}$ with power of $3 \mathrm{~mW} / \mathrm{cm}^{2}$ for $10 \mathrm{~min}$ (Stratalinker 2400, Stragtagene, CA, USA) to directly immobilize the poly (T) poly (C)tagged DNA oligonucleotide on plastic surface without any surface modification [24]. Before conducting the SP-PCR, the chip was washed with $0.1 \times$ SSC for 5 min and rinsed with Milli-Q water and dried in incubator $37^{\circ} \mathrm{C}$. The chip was treated with BSA 2.5 $\mathrm{mg} / \mathrm{mL}$ for $30 \mathrm{~min}$ then rinsed with Milli-Q water and dried in an incubator.

\subsection{Solid phase-PCR}

After immobilization of surface primers, the microchip was bonded and fixed with a gene frame (Thermo Fisher Scientific) to create a $25-\mu \mathrm{L}$ reaction chamber surrounding the solid support primer immobilized SAF array. The PCR master mix was loaded by 
pipette into the gene frame and sealed with a coverslip. The SP-PCR was conducted in a ProFlex $^{\mathrm{TM}} 2 \mathrm{x}$ flat PCR System (Thermo Fisher Scientific) The mastermix contained 2 ng of DNA template, $1 \times$ Phusion $^{\circledR}$ Human Specimen PCR Buffer (Thermo Fisher Scientific), 150 $\mathrm{nM}$ of forward and $1500 \mathrm{nM}$ reverse primers, and $0.05 \mathrm{U} / \mu \mathrm{L}$ Phusion Human Specimen DNA polymerase (Thermo Fisher Scientific). The SP-PCR condition was as follows: $94{ }^{\circ} \mathrm{C}$ for $3 \mathrm{~min}$, followed by 30 cycles of $94^{\circ} \mathrm{C}$ for $20 \mathrm{~s}, 60^{\circ} \mathrm{C}$ for $20 \mathrm{~s}, 72{ }^{\circ} \mathrm{C}$ for $20 \mathrm{~s}$. After the SP-PCR, the chamber was washed with $4 \times$ SSC for 5 min, $0.1 \times$ SSC containing $1 \%$ Tween20 for $5 \mathrm{~min}$, then rinsed with deionized water and dried at room temperature.

\subsection{Data analysis}

The microchip was scanned using a BioAnalyzer 4F/4S scanner with 200-ms shutter time (LaVisionBioTec GmbH, Bielefeld, Germany). Fluorescence intensity was quantified using ImageJ software [25]. A circle was adjusted to the size of the SAF, and the mean value of grey levels of the pixels inside a fluorescent spot was calculated. A square was drawn surrounding the circle, and the mean signal was taken as background 1 . To determine the signal to noise ratio (SNR), the mean signal fluorescent spot with the sample without probe target (NC, negative control) was used as background 2. SNR in this study was defined as the mean signal intensity of the feature subtracted from the mean background 2 and divided by the variation of the background 2 . The limit of detection was determined at SNR of 3. All the experiments were performed in triplicate.

\section{Results and discussion}




\subsection{Structural characterization of 3D structures}

The design of the chip is based on the calculation of the distribution of fluorescence intensity emitted into the polymer for fluorophores on air/water/polymer interfaces as described elsewhere [13]. To select a suitable substrate for fabrication of the disposable chips, one should consider the proportion of the light emitted into the chip part as a function of its refractive index for two interfaces: air/substrate and water/substrate. Moreover, the higher the refractive index of the substrate, the higher the portion of the fluorescent light is emitted into it. Theoretically, these SAF structures can collect light at emission angles of up to $79.2^{\circ}$ and $63.2^{\circ}$ for air/polymer and water/polymer interfaces, respectively (the maximum angle for total internal reflection). So the slope of the SAF structure defines the intensities of the signal generated. The fluorescence intensities collected by using these SAF structures are about $98 \%$ (air/Polymer) and $78 \%$ (water/Polymer) is calculated in comparison to the percentage acquired using a perfect parabolic lens for signal intensity collection[26]. It has been discussed in our previous work that a lower signal is collected if the front face of the SAF structure is used because the light is emitted in all the directions that make the collection of light dependent on the numerical aperture of the optical device (Figure 1A). However, use of rear face of the SAF structure allows us to collect a large portion of the light that was lost in case of collection from the front side. So using the same size of the numerical aperture of the optical system, we can collect more signal, which is required for various low-cost optical detection systems. A graphic sketch of FAPL is shown in Figure 2B. Despite conventional 
method of photolithography, this developed FAPL method is having a controlled angle tilting and motorized rotating chuck to form the slope around the microstructures. Therefore, this rotating motorized stage enables us to persistently keep the tilted angle of chuck and rotation of stage with $15 \mathrm{rmp}$ that allows having exposure to UV light from all side and leads to the slope angles of SAF structures. In our process of fabrication, we achieved 3 different sizes of the SAF structures $(50 \mu \mathrm{m}, 100 \mu \mathrm{m}$, and $150 \mu \mathrm{m})$. In Figure 3, SEM images are shown for all the stages of the fabrication of the L-SAF structures: At the stage after the FAPL $(A, B)$, after electroplating the shim and stripping of the photoresist (C) and, finally, after the chip fabrication by injection molding (D).

In Figure 4, the SEM images for all the three sizes are shown. The SAF structures exhibit uniformity and low surface roughness after the lithography as well as after the injection molding. The optical characterization is presented in the Figure $4 \mathrm{~A}$ for $50 \mu \mathrm{m}$, 4E for $100 \mu \mathrm{m}$, and $4 \mathrm{I}$ for $150 \mu \mathrm{m}$. Rest of the SEM imaging is done after the final stage of the fabrication where SAF structures are replicated on to the polymeric chip after injection molding. Figure 4B, 4C, 4D; represent $50 \mu \mathrm{m}$ size of the SAF structure array, same it continues to Figure 4F, 4G, 4H for $100 \mu \mathrm{m}$ and Figure $4 \mathrm{~J}, 4 \mathrm{~K}, 4 \mathrm{I}$ for $150 \mu \mathrm{m}$. The achieved slope in the SAF structures during the lithography process also helps us out to get the better injection molding and also not to face the problem of polymer deformation during the demolding of the polymer chip. This also reflects in the SEM images of different size of the structures on the polymeric chip, which do not have any defects on the surface. 


\subsection{Comparisons between micro-milled SAF (M-SAF) and lithography made (L-SAF) array}

The SEM images show the apparent difference in between the surface roughness of SAF structures fabricated by FAPL and micro-milling process. As discussed in our previous report, the surface roughness at the sidewall of the SAF structures will give light scattering effects [13]. Since where the fluorescent light is collected using total internal reflection at the surface, roughness will cause a loss of signal. To compare M-SAF and LSAF, the surface roughness of the sidewall surfaces was characterized using a surface profiler (Dektak XTA stylus profiler, Bruker) and Gwyddion 2.20 analysis software as shown in Figure 5. The sidewalls of M-SAF structures (Figure $5 \mathrm{E}$ ) have a high roughness average $\left(R_{a}: 12 \pm 2 \mu \mathrm{m}\right)$. Such average is caused by ring-like structures generated by the finite stepping of the milling bead during the milling process. Moreover, polishing of such small sloped surfaces is a complicated process to perform and can reduce roughness of about $5-10 \%$ compared to the unpolished structures. As can be seen in figure $5 \mathrm{~B}, \mathrm{FAPL}$ process allows the fabrication of SAF structures with extremely low surface roughness $\left(R_{a}: 0.5 \pm 0.1 \mu \mathrm{m}\right)$. It is important to mention that Dektak stylus profiler tip was used to profile directly on the SAF surface of injection molded chip to achieve the real surface morphology than optical profiling.

To compare the LOD between L-SAF and M-SAF array, the same probe (DNA labeled with Cy3 label) with serial dilution (from $1 \mu \mathrm{M}$ to $1 \mathrm{pM}$ ) is used and spotted with same way on front of both types of SAF array. Because of higher roughness, the M-SAF array indicates more background signal than the L-SAF (Figure 6A), which leads to the 
LOD of the L-SAF (100 $\mu \mathrm{m}$ size) is significantly improved in comparing to the M-SAF array

(Figure 6B). The LOD for L-SAF lies between the 0.05 to $0.5 \mathrm{nM}$, corresponding to $6.62 \times 10^{3}$ and $6.62 \times 10^{4}$ molecules, respectively. While the LOD for M-SAF is $10 \mathrm{nM}$ $\left(1.32 \times 10^{6}\right.$ molecules $)$. Therefore, it was clearly observed that L-SAF provided better performance over the M-SAF and fabricated smaller size of L-SAF should be tested and compared with the other size of SAF structures in their signal performance.

\subsection{Limit of detection for different size of SAF array}

To determine the detection limit for the different size of L-SAF array were scanned using a BioAnalyzer 4F/4S scanner with 400-ms shutter time (LaVisionBioTec GmbH, Bielefeld, Germany). The spotting serial dilutions of the probes of DNA labeled with Cy3 label ranging from $1 \mu \mathrm{M}$ to $1 \mathrm{pM}$ on L-SAF structures. To define the best exposure time to find the LOD all the graphs are plotted against the different exposure time and signal to noise ratio (Figure S5 supplementary). The signal at different concentration range was measured from the whole array of SAF from front (Figure 7a) and rear side (Figure 7b). Each concentration is used with the constant volume of $220 \mathrm{pL}$ on each SAF structure. The LOD of the L-SAF array was determined to be as low as $0.05 \mathrm{nM}\left(6.62 \times 10^{3}\right.$ molecules) for $50 \mu \mathrm{m}, 0.5 \mathrm{nM}\left(6.62 \times 10^{4}\right.$ molecules $)$ for $100 \mu \mathrm{m}$, and $10 \mathrm{nM}\left(1.32 \times 10^{6}\right.$ molecules) for $150 \mu \mathrm{m}$ SAF structure at the constant exposure time for all size of L-SAF array. If we compare the different size of the L-SAF array with its detection limit the smaller size $(50 \mu \mathrm{m})$ shows higher signal intensity at per $\mu \mathrm{m}^{2}$ area (Figure $\mathrm{S} 5$, in the supplementary data). The linear rage of the signal detection lies between the 
concentration ranges from $0.05 \mathrm{nM}$ to $1 \mu \mathrm{M}$ then signal starts to get saturated due to a high exposure time. It is also important to mention here that the small volume of the sample is sufficient to cover the all front surface of the smaller size of the L-SAF surface to give the better signal. However, if the sample volume is increased up to the volume when it is sufficient to completely cover the front face surface of SAF with bigger size (100 and $150 \mu \mathrm{m})$, the LOD for these size can be further improved with higher volume.

\subsection{SP-PCR on fabricated SAF structures}

As mentioned in our previous reports $[18,27,28]$, the SP-PCR has become increasingly popular for molecular diagnosis integrated with lab-on-a-chip devices. In this study, we addressed a number of advantages of the small size of the SAF structure (50 um) with fabricated by free angle lithography process compared to the bigger size as well as SAF structure from the conventional process. To test the applicability of these 3D SAF structure for further biomedical application, we carried out the SP-PCR on this SAF structure embedded in a microchip. The surface primers were spotted on the front of SAF array as indicated in Figure 8. The DNA target gene (pneumolysin gene) from Streptococcus pneumonia was specifically amplified using SP-PCR on the front of the LSAFs on chip. Where were modified with surface primers for DNA target gene. As showed in the figure $8 \mathrm{~b}$, it shows the high fluorescent signal and there are no any fluorescent signals observed containing non-specific primers. Since there was not any specific primer available so no amplification took place during SP-PCR reaction as presented in Figure 8b. 


\section{Conclusions}

In this paper, we have presented a novel free angle lithography method to make a disposable chip with a miniaturized $(50 \mu \mathrm{m})$ L-SAF array, with the successful SP-PCR for selective and sensitive detection of pathogen. The intensity of the signal vs. probe concentration measured from the rear face for different size of SAF structures and defined the LOD (0.05 $\mathrm{nM})$ of the smallest SAF structure. The use of such small size of SAF structure will enable us to use less volume (220 pL) and save expensive primer and molecular probes in an experiment. The fabrication of SAF structure is important to achieve the small size and higher density of the SAF on a chip. Despite the complication of the FAPL process for fabrication of SAF structures it still embraces advantages of lower surface roughness and background signal noise (Figure 6A). The presented smaller SAF have great potential to be used for the multiple detections in a small area with high sensitivity and large field of view which can help to use such chip without a sophisticated optical system. This will allow us to make a cost-effective experimental design with a low volume of the sample and optical readout system. In future development, a lower detection limit could be achieved by reducing the background signal and by using higher wavelength fluorophores. The combination of SP-PCR and L-SAF array would be widely used as a high-throughput biosensor to analyze food, clinical and environmental samples. 


\section{Acknowledgements}

This work was financially supported by the European Union's Horizon 2020 research and Innovation program, grant agreement No. 687697.

\section{References}

[1] U.M. Attia, S. Marson, J.R. Alcock, Micro-injection moulding of polymer microfluidic devices, Microfluid. Nanofluidics 7 (2009) 1-28. http://dx.doi.org/10.1007/s10404-009-0421-x.

[2] S.H. Choi, D.S. Kim, T.H. Kwon, Microinjection molded disposable microfluidic lab-on-a-chip for efficient detection of agglutination, Microsyst. Technol. 15 (2009) 309-316. https://doi.org/10.1007/s00542-008-0689-x.

[3] S. Tasoglu, H. Cumhur Tekin,F. Inci, S. Knowlton, S.Q. Wang, F. Wang-Johanning, G. Johanning, D. Colevas, U. Demirci, Advances in Nanotechnology and Microfluidics for Human Papillomavirus Diagnostics, Proc. IEEE 103 (2015) 161-178. https://doi.org/10.1109/JPROC.2014.2384836.

[4] G.S. Fiorini, D.T. Chiu, Disposable microfluidic devices: Fabrication, function, and application, Biotechniques 38 (2005) 429-446. https://doi.org/10.2144/05383RV02.

[5] I.R.G. Ogilvie, V.J. Sieben, B. Cortese, M.C. Mowlem, H. Morgan, Chemically resistant microfluidic valves from Viton ${ }^{\circledR}$ membranes bonded to COC and PMMA, Lab Chip 11 (2011) 2455-2459. https://doi.org/10.1039/c1lc20069k.

[6] G. Khanarian, Optical properties of cyclic olefin copolymers, Opt. Eng. 40 (2001) 1024-1029. 
https://doi.org/10.1117/1.1369411.

[7] T.C.K. Yang, S.H.Y. Tsai, S.F. Wang, C.C. Juan, Dielectric and thermal studies of inorganic microfillers on polymer microwave substrates - Metallocene cyclic olefin copolymers (COC), Compos. Sci. Technol. 62 (2002) 655-661. https://doi.org/10.1016/S0266-3538(02)00014-3.

[8] H. Klank, J.P. Kutter, O. Geschke, $\mathrm{CO}(2)$-laser micromachining and back-end processing for rapid production of PMMA-based microfluidic systems, Lab Chip 2 (2002) 242-246. https://doi.org/10.1039/b206409j.

[9] T.F. Hong, W.J. Ju, M.C. Wu, C.H. Tai, C.H. Tsai, L.M. Fu, Rapid prototyping of PMMA microfluidic chips utilizing a $\mathrm{CO}_{2}$ laser, Microfluid. Nanofluidics 9 (2010) 1125-1133. https://doi.org/10.1007/s10404-010-0633-0.

[10] Ogilvie, I.R.G., Sieben, V.J., Floquet, C.F.A., Zmijan, R., Mowlem, M.C., Morgan, H., 2010. Reduction of surface roughness for optical quality microfluidic devices in PMMA and COC. J. Micromech. Microeng. 20, 65016. https://doi.org/10.1088/0960-1317/20/6/065016.

[11] Campo, A. del., Greiner, C., 2007. SU-8: a photoresist for high-aspect-ratio and 3D submicron lithography. J. Micromech. Microeng. 17, R81. https://doi.org/10.1088/09601317/17/6/R01.

12. Lorenz, H., Despont, M., Fahrni, N., LaBianca, N., Renaud, P., Vettiger, P., 1997. SU-8: a lowcost negative resist for MEMS. J. Micromech. Microeng. 7, 121. https://doi.org/10.1088/0960-1317/7/3/010. 
[13] T.Q. Hung, Y. Sun, C.E. Poulsen, T. Linh-Quyen, T, W.H. Chin, D.D. Bang, A. Wolff, Miniaturization of a micro-optics array for highly sensitive and parallel detection on an injection moulded lab-on-a-chip, Lab Chip 15 (2015) 2445-2451. https://doi.org/10.1039/C5LC00176E.

[14] Tanzi, S., Ostergaard, P.F., Matteucci, M., Christiansen, T.L., Cech, J., Marie, R., Taboryski, R., 2012. Fabrication of combined-scale nano- and microfluidic polymer systems using a multilevel dry etching, electroplating and molding process. J. Micromech. Microeng. 22, 115008. https://doi.org/10.1088/0960-1317/22/11/115008.

[15] C.M. Winterflood, T. Ruckstuhl, S. Seeger, Fast and sensitive interferon- $\gamma$ assay using supercritical angle fluorescence, Biosensors 3 (2013) 108-115. https://dx.doi.org/10.3390\%2Fbios3010108.

[16] T. Ruckstuhl, D. Verdes, Supercritical angle fluorescence (SAF) microscopy, Opt. Express 12 (2004) 4246-4254. https://doi.org/10.1364/OPEX.12.004246.

[17] K.A. Wolfe, M.C. Breadmore, J.P. Ferrance, M.E. Power, J.F. Conroy, P.M. Norris, J.P. Landers, Toward a microchip-based solid-phase extraction, Electrophoresis 23 (2002) 727733. https://doi.org/10.1002/1522-2683(200203)23:5\%3C727::AID-ELPS727\%3E3.0.CO;2-O.

[18] Y. Sun, R. Dhumpa, D.D. Bang, J. Høgberg, K. Handberg, A. Wolff, A lab-on-a-chip device for rapid identification of avian influenza viral RNA by solid-phase PCR, Lab Chip 11 (2011) 14571463. https://doi.org/10.1039/c0lc00528b. 
[19] Sato, H., Matsumura, H., Keino, S., Shoji, S., 2006. An all SU-8 microfluidic chip with built-in 3D fine microstructures. J. Micromech. Microeng. 16, 2318. https://doi.org/10.1088/09601317/16/11/010.

[20] Joye, C.D., Calame, J.P., Garven, M., Levush, B., 2010. UV-LIGA microfabrication of $220 \mathrm{GHz}$ sheet beam amplifier gratings with SU-8 photoresists. J. Micromech. Microeng. 20, 125016. https://doi.org/10.1088/0960-1317/20/12/125016.

[21] F. Pérennès, M. Matteucci, W. Jark, B. Marmiroli, Fabrication of refractive X-ray focusing lenses by deep X-ray lithography, Microelectronic Engineering 78-79 (2005) 79-87. https://doi.org/10.1016/j.mee.2004.12.012.

[22] Stormonth-Darling, J.M., Pedersen, R.H., How, C., Gadegaard, N., 2014. Injection moulding of ultra high aspect ratio nanostructures using coated polymer tooling. J. Micromech. Microeng. 24, 75019. https://doi.org/10.1088/0960-1317/24/7/075019.

[23] O. Greiner, P.J. Day, P.P. Bosshard, F. Imeri, M. Altwegg, D. Nadal, Quantitative detection of Streptococcus pneumoniae in nasopharyngeal secretions by real-time PCR, J. Clin. Microbiol. 39 (2001) 3129-3134. https://doi.org/10.1128/JCM.39.9.3129-3134.2001.

[24] Y. Sun, I. Perch-Nielsen, M. Dufva, D. Sabourin, D. D. Bang, J. Høgberg, A. Wolff, Direct immobilization of DNA probes on non-modified plastics by UV irradiation and integration in microfluidic devices for rapid bioassay, Anal. Bioanal. Chem. 402 (2012) 741-748. https://doi.org/10.1007/s00216-011-5459-4. 
[25] C.A. Schneider, W.S. Rasband, K.W. Eliceiri, NIH Image to ImageJ: 25 years of image analysis, Nat. Methods 9 (2012) 671-675. https://doi.org/10.1038/nmeth.2089.

[26] T. Ruckstuhl, M. Rankl, S. Seeger, Highly sensitive biosensing using a supercritical angle fluorescence (SAF) instrument, Biosens. Bioelectron. 18 (2003) 1193-1199. https://doi.org/10.1016/S0956-5663(02)00239-7.

[27] T.Q. Hung, W.H. Chin, Y. Sun, A. Wolff, D.D. Bang, A novel lab-on-chip platform with integrated solid phase PCR and supercritical angle fluorescence (SAF) microlens array for highly sensitive and multiplexed pathogen detection, Biosens. Bioelectron. 90 (2016) 217223. https://doi.org/10.1016/j.bios.2016.11.028.

[28] W.H. Chin, Y. Sun, J. Høgberg, T.Q. Hung, A. Wolff, D.D. Bang, Solid-phase PCR for rapid multiplex detection of Salmonella spp. at the subspecies level, with amplification efficiency comparable to conventional PCR, Anal. Bioanal. Chem. 409 (2017) 2715-2726. https://doi.org/10.1007/s00216-017-0216-y. 


\section{Biographies}

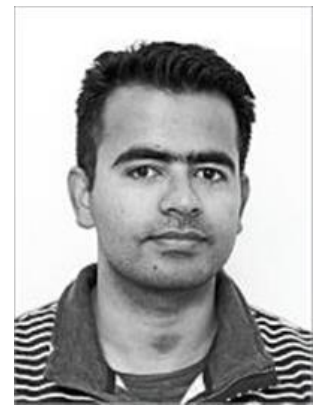

Krishna Kant

Dr. Krishna Kant is a postdoctoral research fellow at Technical University of Denmark (DTU) and working on the development of Point of care (POC) diagnostic system in the detection of Sepsis disease under the Horizon 2020, SMARTDIAGNOS project. During his career, he received various fellowships (Lady Davis fellow, Israel, ARC (Australian research council) fellowship, etc.) expertise in the field of nanomaterials and microfluidics. He obtained his Master's degree in Nano-Biotechnology from Amity University, Noida, India, and Ph.D. in the field of electrochemical bio-sensing form Flinders University, Adelaide, Australia. So far in his research carrier, Dr. Kant published various research articles in reputed international journals (Biotechnology Advance, Biomaterial, Biosensor Bioelectronics, Analyst, Electrochimica Acta, etc.)

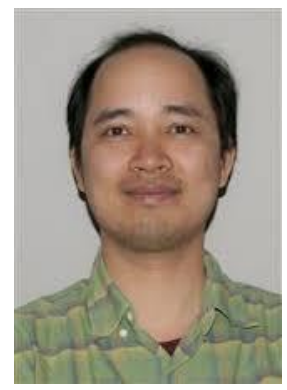

\section{Tien Anh Ngo}

Dr. Tien Anh Ngo received his Ph.D. in Fundamental Energy Science for emerging molecular design principles of functional biomolecules and biomolecular assemblies in Division of Biofunctional Chemistry Research Section, Institute of Advanced Energy-Kyoto University with Prof. Takashi Morii (Japan, Mar. 2015), followed by postdoctoral research (2015-2016) with the same group. Since April. 2016, he joined Research Group for Analytical and Predictive Microbiology, LAMINATE team with Prof. D.D. Bang at Technical University of Denmark, National Food Institute (DTU Food) as a postdoc. His area of research is the development of Molecular Diagnostics assays with particular emphasis on the development of a Lab-on-a-chip platform integrated solid phase PCR for multiple detection pathogens, microbial resistance genes, and microRNAs for Sepsis Diagnosis and detection technologies for point of care industries. 


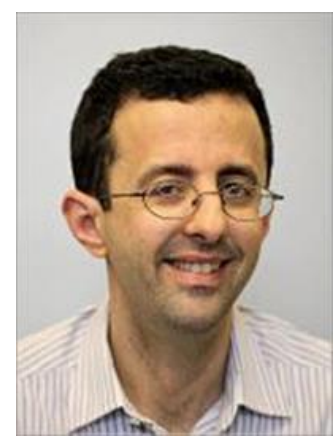

\section{Marco Matteucci}

Dr. Marco Matteucci a senior researcher at Technical University of Denmark (DTU) and working on the micro and nano fabrication of microfluidic chip for the development of Point of care (POC) diagnostic system. Marco was involved in the project dedicated to SERS based Sensing and Centrifugal Microfluidics. He is holding the expertise in the lithography based procedure and development of polymer chips using Injection molding. Marco completed his $\mathrm{PhD}$ from Università degli Studi di Trieste 2007.

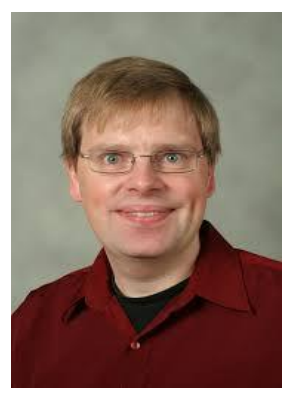

\section{Anders Wolff}

Dr. Anders Wolff received his MSc in Chemical Engineering from Technical University of Denmark (DTU) in 1993 and Ph.D. in Biochemical and Engineering from the Delft University of Technology, the Netherlands in Dec 1997. In 1998, Dr. Wolff joined the Department of Micro and Nanotechnology (now DTU Nanotech, DTU, Lyngby, Denmark). In 2000, he was appointed as associate professor and leaded the Cell Handling Group (now BioLabChip) at DTU. His research interests are PCR chip with integrated heaters and thermal sensor, integrated microsystem for sample preparation and DNA amplification. Dr. Wolff owned seven patents, 120 papers published in international scientific journals. 


\section{List of figures}

Figure 1. Schematic representation of the fabrication of 3D SAF structures using FAPL and transfer of SAF structure to the polymeric chip by injection molding (A) and SP-PCR on fabricated SAF for nucleic acid detection on Chip (B).

Figure 2. Schematic representation of fabrication steps of L-SAF array using FAPL process. Spin coating of photoresist and edge bead removal is presented in $(A)$, and free angle UV exposure over the rotatory chuck and development of the exposed Si is presented in (B), the transfer of the fabricated 3D L-SAF array to the polymeric chip. Electroplating of the seed layer of NiV (80nm) and thick layer (350 $\mu \mathrm{m}$ ) of Ni for making a shim for injection molding is presented in (C).

Figure 3. Characterization of fabricated SAF array using FAPL process using SEM. (A) SAF structures made in photoresist, (B) 3D view of the same SAF structure inset scale is $20 \mu \mathrm{m},(C) \mathrm{Ni}$ shim made after electroplating, (D) SAF structure on injection molded polymer chip.

Figure 4. Characterization of fabricated SAF array using FAPL process using optical microscope and SEM. $(A, E, I)$ are optical images of the SAF structure array. SEM images on the polymeric chip after transfer of SAF structure by injection molding, for $50 \mu \mathrm{m}$ size $(B, C, D)$, for $100 \mu \mathrm{m}(F$, $G, H)$ and for $150 \mu m(J, K, L)$.

Figure 5. SEM images of the SAF structures on polymeric chip after injection molding $(A$, B) FAPL fabricated SAF (L-SAF: $100 \mu M)$, (D, E) micro-milling fabricated SAF (M-SAF: 100 $\mu M)$, surface profile and average surface roughness of L-SAF (C) and M-SAF (F). 
Figure 6. (A) Comparison of the background signal from L-SAF and M-SAF, (B) Graph for the mean value of fluorescence for L-SAF and M-SAF spotted with different number of fluorescent molecules after subtracting background. The spotting volume was $220 \mathrm{pL}$ and exposure time $400 \mathrm{~ms}$ for the same size of L-SAF and M-SAF.

Figure 7. Fluorescent images of L-SAF structures, spotted with 220 pl Cy3-labeled DNA probe ranging from $1 \mathrm{pM}\left(1.32 \times 10^{2}\right.$ molecules $/ \mathrm{SAF}$ structure) to $1 \mu \mathrm{M}\left(1.32 \times 10^{8}\right.$ molecules/SAF structure). The images are taken from A, front side (no use of SAF optical structure) and B, rear side (use of SAF optical structure). In B, the light ring corresponded to the emission reflected at the side wall of the L-SAF structure. Bottom two graphs: signal to noise ratio from 50, 100, and 150 Mm-L-SAF arrays scanned by using BioAnalyzer 4F/4S scanner at constant exposure time (400 micro sec) and with different number of fluorescent molecules per SAF structure. Left graph: scanned from the front side (no use of SAF optical structure), and right graph: scanned from the rear side (use of SAF optical structure).

Figure 8. SP-PCR on the L-SAF array. (A) Microarray layout. The surface primers for specific amplification of DNA target gene from Streptococcus pneumonia and Streptococcus spp and Cy3 labeled-poly $T$ (10) C (10) as a positive DNA probe were immobilized on the front of L-SAF for SP-PCR reaction. (B) Fluorescent scanning image of the L-SAF array after SP-PCR. 
Figure 1. Kant. et. al.

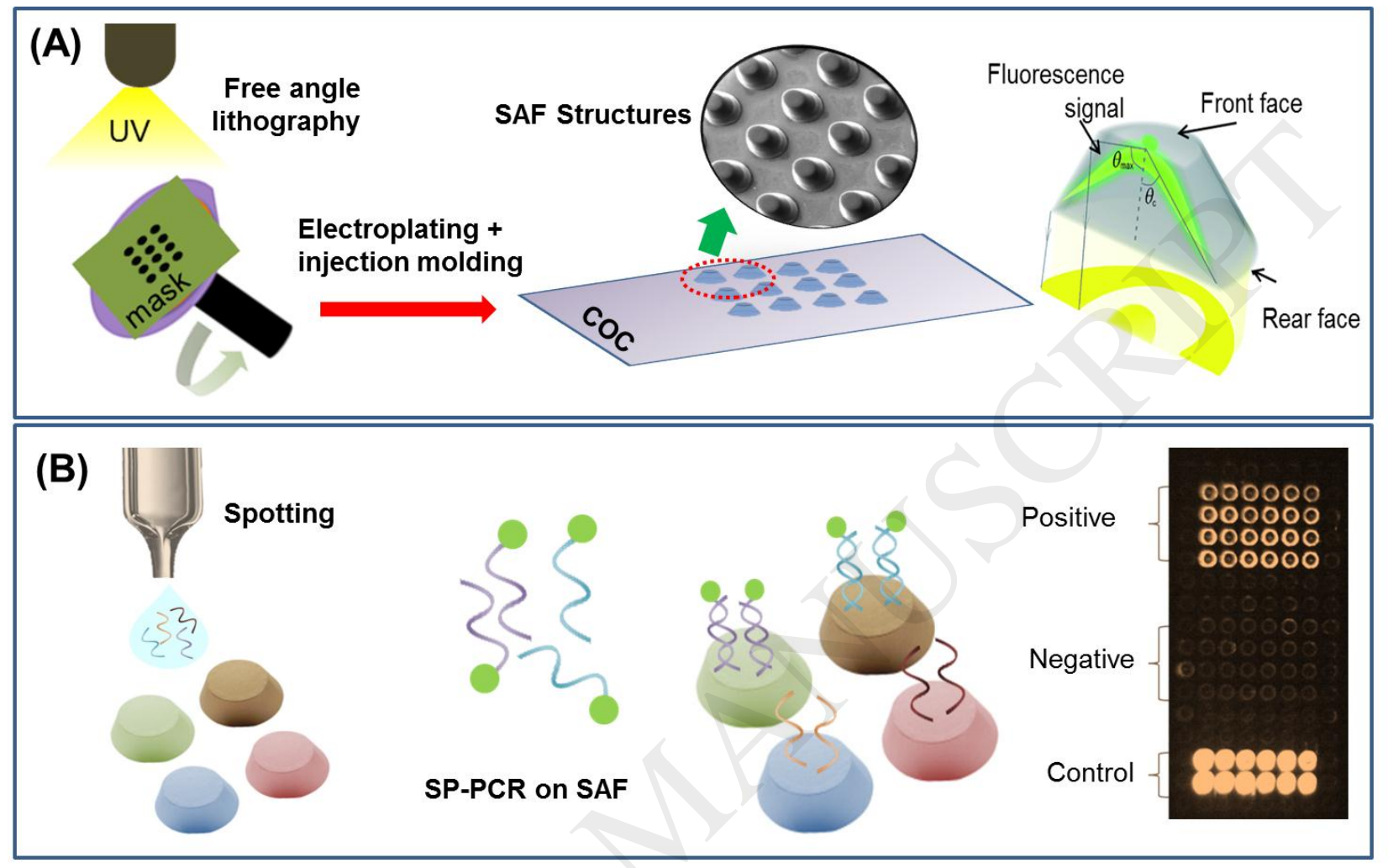

Schematic representation of the fabrication of 3D SAF structures using FAPL and transfer of SAF structure to the polymeric chip by injection molding (A) and SP-PCR on fabricated SAF for nucleic acid detection on Chip. 
Figure 2. Kant. et. al.
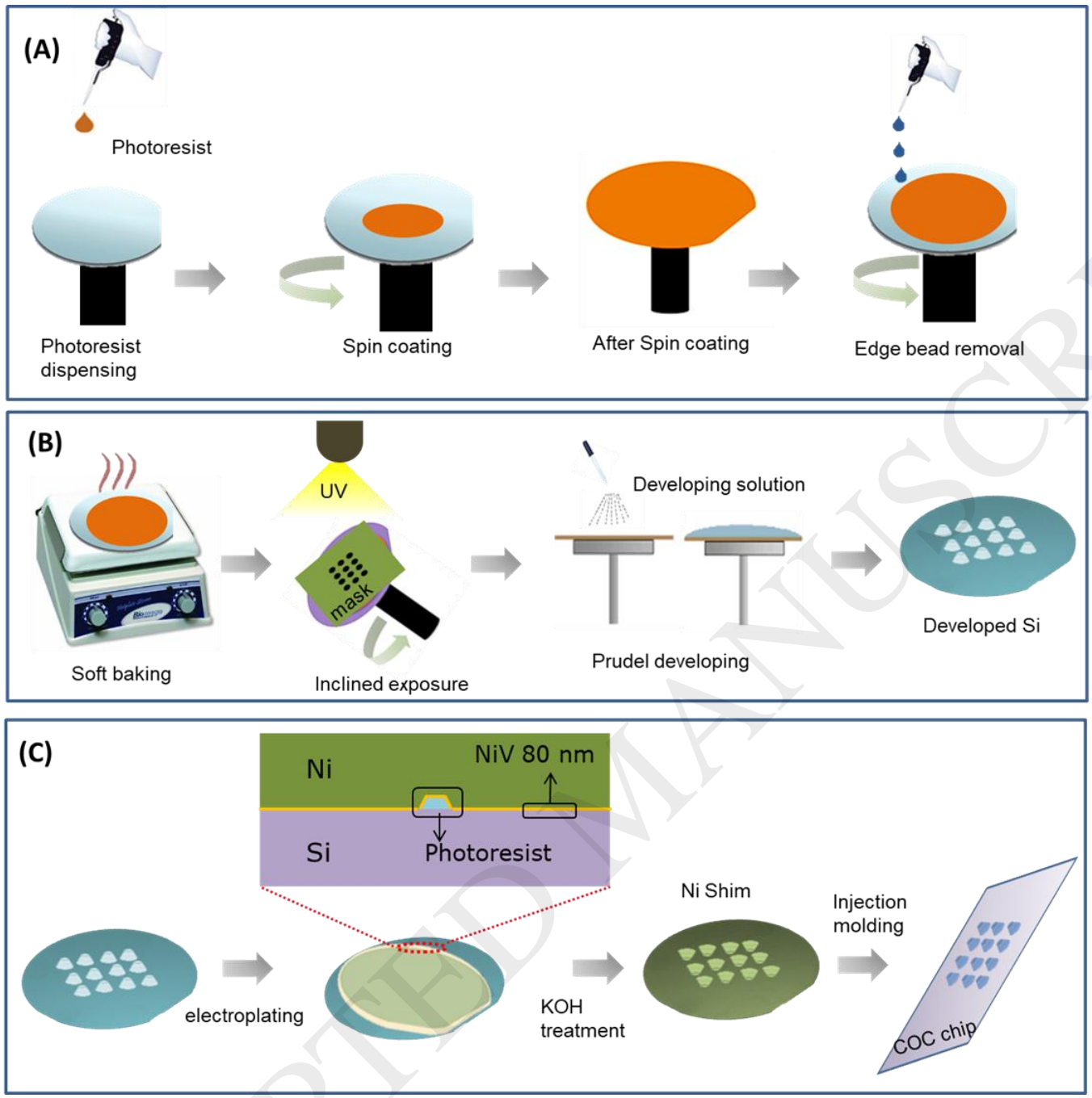

Schematic representation of fabrication steps of L-SAF array using FAPL process. Spin coating of photoresist and edge bead removal is presented in (A), and free angle UV exposure over the rotatory chuck and development of the exposed Si is presented in (B), the transfer of the fabricated 3D L-SAF array to the polymeric chip. Electroplating of the seed layer of NiV (80nm) and thick layer (350 $\mu \mathrm{m}$ ) of Ni for making a shim for injection molding is presented in (C). 
Figure 3. Kant. et. al.
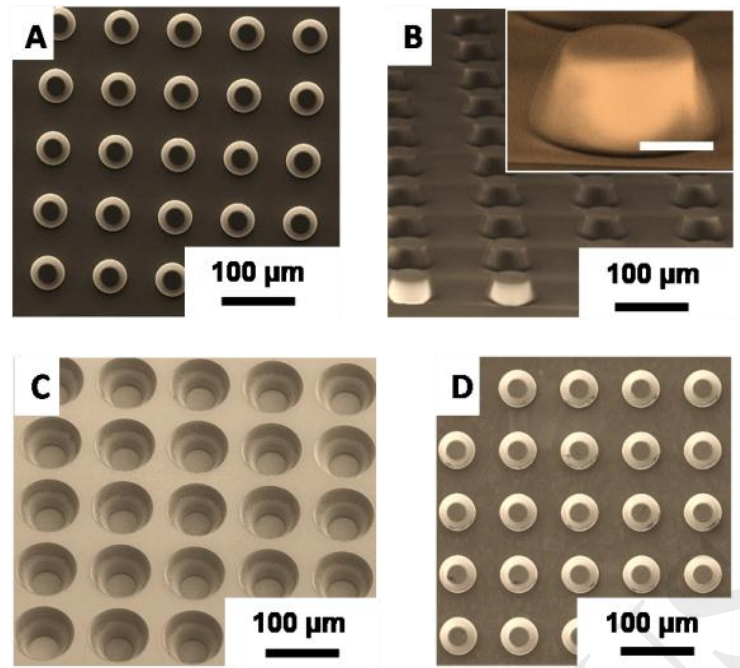

Characterization of fabricated SAF array using FAPL process using SEM. (A) SAF structures made in photoresist, (B) 3D view of the same SAF structure inset scale is $20 \mu \mathrm{m},(C)$ Ni shim made after electroplating, (d) SAF structure on injection molded polymer chip. 
Figure 4. Kant. et. al.

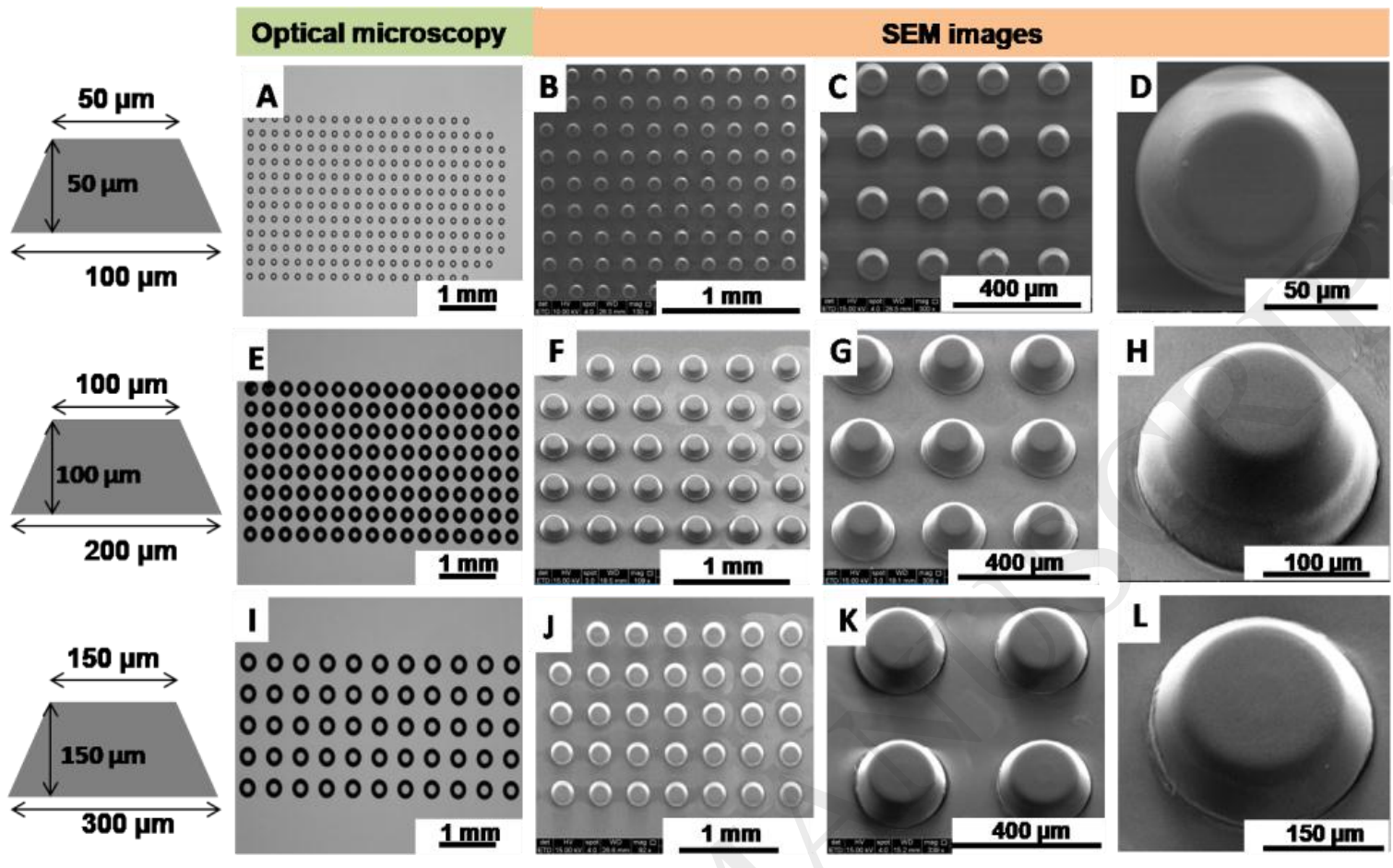

Characterization of fabricated SAF array using FAPL process using optical microscope and SEM.

$(A, E, I)$ are optical images of the SAF structure array. SEM images on the polymeric chip after transfer of SAF structure by injection molding, for $50 \mu \mathrm{m}$ size $(B, C, D)$, for $100 \mu \mathrm{m}(F, G, H)$ and for $150 \mu m(J, K, L)$. 
Figure 5. Kant. et. al.
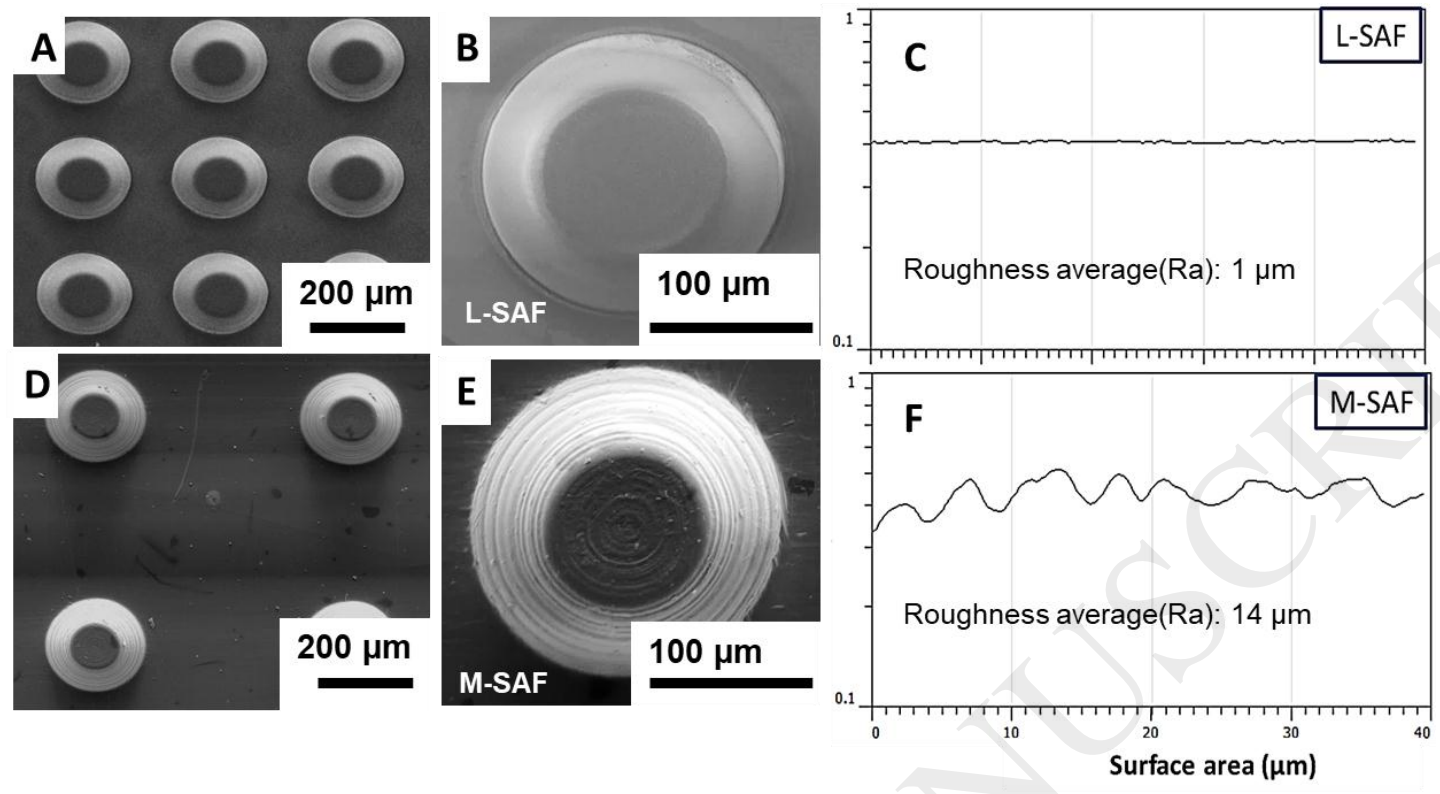

SEM images of the SAF structures on polymeric chip after injection molding $(A, B) F A P L$ fabricated SAF (L-SAF: $100 \mu \mathrm{M})$, (D, E) micro-milling fabricated SAF (M-SAF: $100 \mu M)$, surface profile and average surface roughness of L-SAF (C) and M-SAF (F). 
Figure 6. Kant. et. al.

A

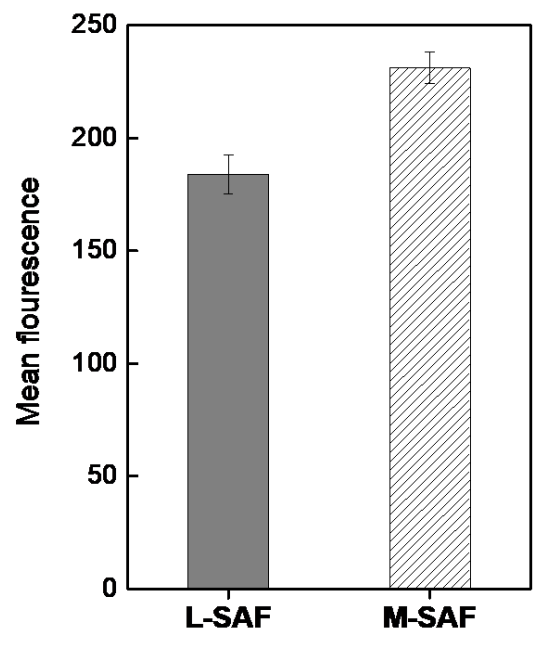

B

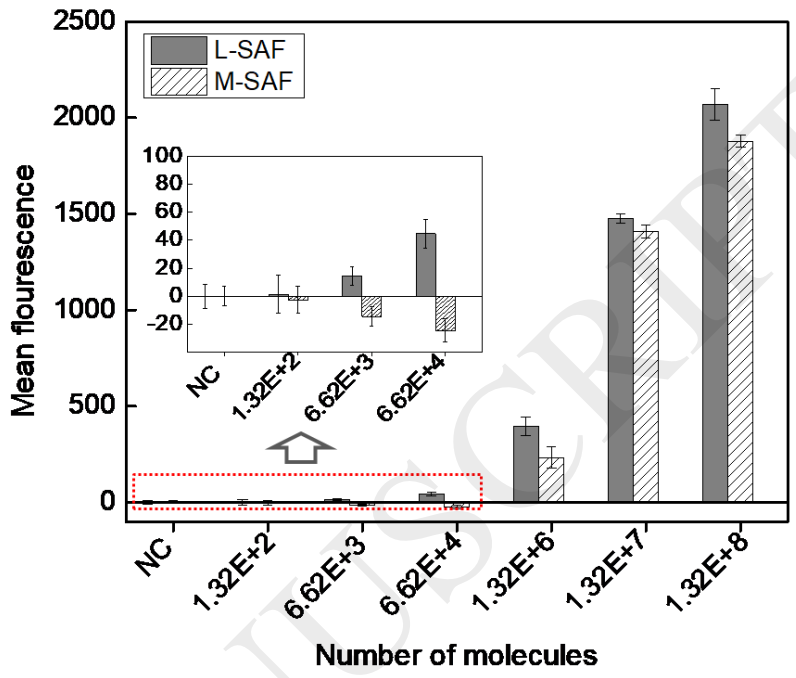

(A) Comparison of the background signal from L-SAF and M-SAF, (B) Graph for the mean value of fluorescence for L-SAF and M-SAF spotted with different number of fluorescent molecules after subtracting background. The spotting volume was $220 \mathrm{pL}$ and exposure time $400 \mathrm{~ms}$ for the same size of L-SAF and M-SAF. 
Figure 7. Kant. et. al.

A
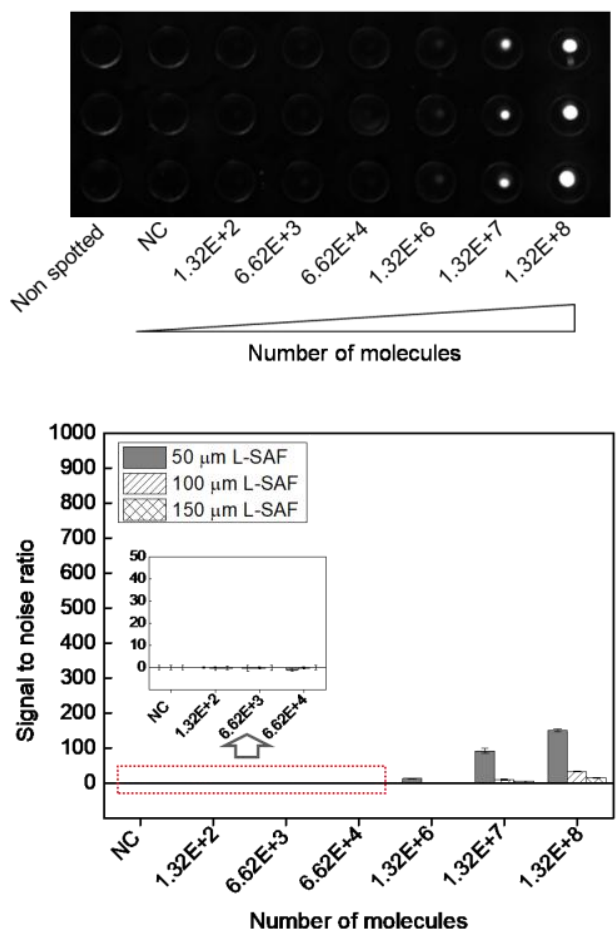
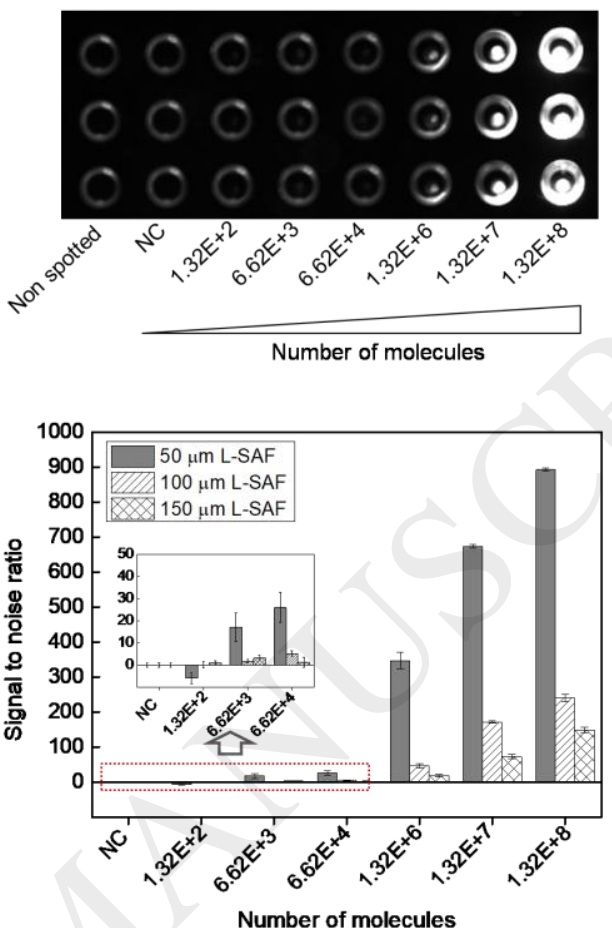

Fluorescent images of L-SAF structures, spotted with 220 pl Cy3-labeled DNA probe ranging from $1 \mathrm{pM}\left(1.32 \times 10^{2}\right.$ molecules/SAF structure) to $1 \mu \mathrm{M}\left(1.32 \times 10^{8}\right.$ molecules $/ \mathrm{SAF}$ structure). The images are taken from A, front side (no use of SAF optical structure) and $B$, rear side (use of SAF optical structure). In $B$, the light ring corresponded to the emission reflected at the side wall of the L-SAF structure. Bottom two graphs: signal to noise ratio from 50, 100, and $150 \mu m$-L-SAF arrays scanned by using Bio-Analyzer 4F/4S scanner at constant exposure time (400 micro sec) and with different number of fluorescent molecules per SAF structure. Left graph: scanned from the front side (no use of SAF optical structure), and right graph: scanned from the rear side (use of SAF optical structure). 
Figure 8. Kant. et. al.

(A)

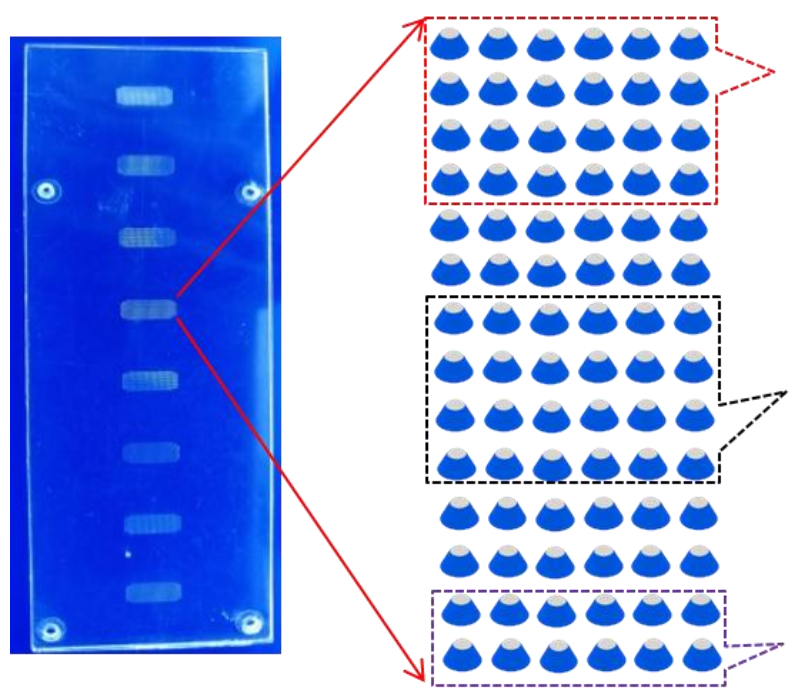

(B)

Strep. pneumonia

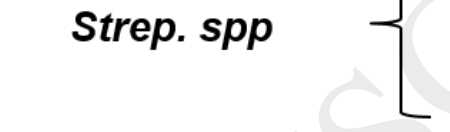

Cy3 labeled-Poly $T(10) C(10)$

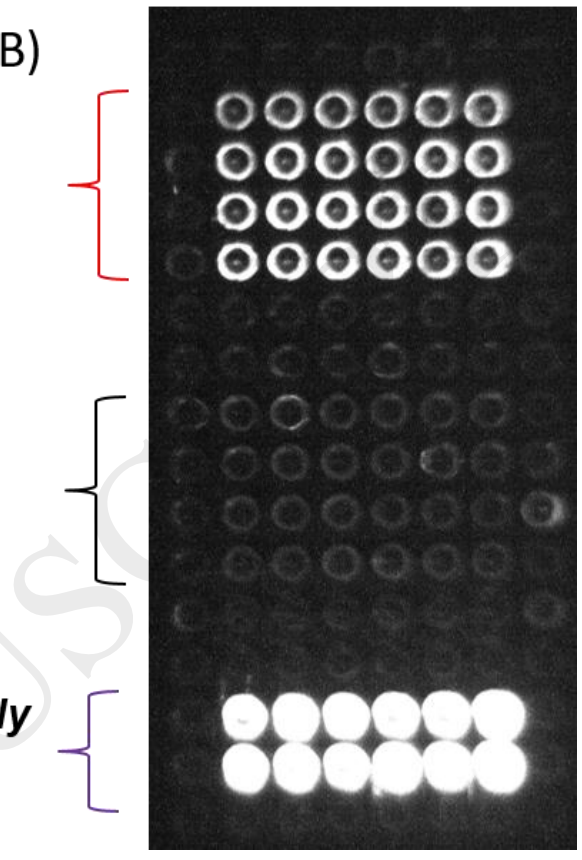

SP-PCR on the L-SAF array. (A) Microarray layout. The surface primers for specific amplification of DNA target gene from Streptococcus pneumonia and Streptococcus spp and Cy3 labeled-poly $T(10) C(10)$ as a positive DNA probe were immobilized on the front of L-SAF for SP-PCR reaction. (B) Fluorescent scanning image of the L-SAF array after SPPCR. 